\title{
Experimental Assessment of a Micro-Mechanical Model for the Static Strength of
} Hybrid Friction-Bonded Interfaces

\author{
D. Castagnetti ${ }^{1}$, E. Dragoni ${ }^{1}$ \\ ${ }^{1}$ Department of Engineering Sciences and Methods, Univ. of Modena and Reggio Emilia, \\ 42122 Reggio Emilia (RE) - Italy
}

\begin{abstract}
Anaerobic adhesives are thermosetting acrylic polymers commonly used to improve the performance of most metal joints. Researches on the static strength of hybrid joints, available in the technical literature, show scanty and contradictory results that do not explain the effect of anaerobic adhesive on the hybrid joint behaviour. An early study by one of the authors of the present study formulates a micro-mechanical model describing the shear power of anaerobic adhesives as a function of the intimate properties of adherends and adhesive at the interface. According to the micro-mechanical model, the high local pressure acting on the thin film of adhesive trapped between the crests of the mating surfaces improves the film shear strength upon the adhesive's at zero pressure. The present work aims to assess this micro-mechanical model through a systematic experimental campaign. The tests are conducted on simple tubular specimens and consider three variables over two levels: adhesive type (weak and strong anaerobic), pressure level during polymerization (0.5 $\mathrm{MPa}$ and $134 \mathrm{MPa})$ and pressure level during failure test (0.5 $\mathrm{MPa}$ and
\end{abstract}


$134 \mathrm{MPa}$ ). The results confirm the proposed micro-mechanical model, and highlight that shear strength slightly differs by applying pressure before or after polymerization.

\section{Keywords}

Anaerobic, friction, mechanical properties of adhesives, hybrid interface, shear strength, micro-mechanical model. 


\section{Introduction}

Anaerobic adhesives are thermosetting acrylic polymers; which are introduced in most metal joints, in particular those relying on mechanical tightening, to obtain efficient friction-bonded interfaces. Bolted joints, flanged couplings or interference fits are typical examples.

In the technical literature, many researches investigate the strength of hybrid joints [1]-[14] but scanty and contradictory experimental results are presented. In [1], O'Reilly develops a design tool for bonded cylindrical joints. In [2], Mahon presents joint design parameters, test results and calculation techniques for bonded friction couplings, with particular reference to automotive drivetrain applications. Romanos [3] provides information on static and fatigue strength of friction bonded interfaces produced using appropriate industrial assembly techniques. Bartolozzi et al. [4] show that the strength of hybrid joints is affected by various factors such as, for example, the coupling pressure. In [5], Croccolo et al. evaluate the residual strength in drive-fit and adhesively bonded cylindrical joints under a tension-tension fatigue cycle and observe a strongly different mechanical behaviour between steel-steel couplings and the aluminium-steel ones. Sekercioglu et al. [6]-[8] investigate the effect of interference fit, bonding clearances, surface roughness, adherent and temperature on the static and dynamic strength of adhesively bonded cylindrical components. Aronovich et al. [9] review the properties of anaerobic adhesive materials during testing and their applications in cylindrical coaxial joints. Mengel et al. [10] show that hydrostatic pressure on adhesive during the curing process significantly increases the strength of the joint. In [11] and [12], Oinonen et al. presents an experimental assessment 
of quasi-static shear strength of epoxy adhesive reinforced steel interfaces and relying on these results develop a damage model for shear decohesion analysis. In [13], Dragoni and Mauri assess the friction and the adhesive contributions to the overall strength of an annular friction bonded interface.

On the basis of this literature review it appears that the effect of anaerobic adhesives on the static strength of hybrid interfaces is not clearly assessed. A classical interpretation is that clamping pressure applied to the surfaces of the hybrid interface produces a direct contact between the metal crests of roughness, confining the adhesive in the gaps between. Therefore, the total shear strength of the hybrid interface build the adhesive shear strength upon those originated by friction.

By relying on a preliminary experimental campaign, Dragoni and Mauri [14] propose a micro-mechanical model (Appendix 1) to describe the shear power of anaerobic adhesives. According to this model, the shear strength depends on the intimate properties of adherends and adhesive at the interface. In particular, the high local pressure acting on the thin film of adhesive trapped between the crests of the mating surfaces improves the film shear strength upon the adhesive's at zero pressure. Stronger adhesives exhibit a higher increase than weaker adhesives for a given local pressure.

The aim of this work is to assess experimentally this micro-mechanical model through a experimental campaign in which the relevant set-up variables are combined systematically. A systematic experimental campaign was performed, by using simple tubular butt-bonded specimens, which originate a uniform normal and shear stress distribution. Three variables 
over two levels are considered: the adhesive type (weak and strong anaerobic), the pressure level during polymerization (low or high) and the pressure level during failure test (low or high).

The results show that the interface cumulative strength significantly increases as the contact pressure increases, regardless of whether the contact pressure is applied before or after polymerization. These results confirm the micro-mechanical model proposed in [13]-[14]. 


\section{Method}

Figure 1a shows the sketch of the tubular adherend that is was used to build the specimens for the experimental tests. The adherends were machined on a lathe, starting from grinded bars, made of normalized mild steel (C40), and having the diameter reported in Figure 1a. The specimen is was obtained by butt-bonding a couple of adherends on the annular surface (inner diameter $16 \mathrm{~mm}$, outer diameter $22 \mathrm{~mm}$ ) shown in the close-up of Figure $1 \mathrm{~b}$. The dimensions of this bonded area come from the maximum axial force $(25 \mathrm{kN})$ and torque (200 Nm) that can be applied by the servo-hydraulic testing machine (MTS MiniBionix 858, Eden Prairie, MN, USA), available in our laboratory. For this bonding surface, the applied pressure under the maximum axial force is about $134 \mathrm{MPa}$. Due to this clamping pressure, the real area of contact is about $8 \%$ of the nominal contact area [15].

Table 1 collects the three variables of the two-level full factorial plan. A weak anaerobic adhesive (Loctite 243 [16], from Henkel, Milano, Italy) and a strong anaerobic (Loctite 638 [17], from Henkel, Milano, Italy) were considered. The polymerization of the adhesive was performed at two clamping pressure levels: $0.5 \mathrm{MPa}$ (given by an axial load of $90 \mathrm{~N}$ ) and $134 \mathrm{MPa}$ (corresponding to an axial load of $24 \mathrm{kN}$ ). Finally, the torque test up to failure was performed at the same nominal pressure levels used for the polymerization phase. On the one hand, the lower nominal pressure value was chosen in order to enforce a uniform and complete contact between the bonding surfaces. On the other hand, the higher nominal pressure value comes from the maximum axial force that the testing machine can apply. In addition to these configurations, a dry specimen (i.e. not bonded) subject to the higher nominal pressure level was considered, in order to have a reference value for the case of a 
pure friction interface. For each specimen configuration three replicates were performed, giving a total of 27 tests.

The preparation of the bonded specimens was done according to the following steps:

1. manual abrasion of the bonding surface with sandpaper (P120);

2. cleaning the adherends with Loctite 7063 degreaser;

3. measurement of the roughness of the bonding surface, with a Hommelwerke electronic rugosimeter (Lamone, Switzerland);

4. mounting of the adherends on the grips of the testing machine;

5. wrapping of the adherends with black adhesive tape to increase heat absorption (Figure 2);

6. measurement of the contact area under an axial load of $1 \mathrm{kN}$ and $24 \mathrm{kN}$ (applied at 5 $\mathrm{kN} / \mathrm{min}$ ) through a layer of tissue paper facing with one of carbon paper;

7. separation of the adherends and removal of the measurement paper of step 6 ;

8. application of the anaerobic adhesive;

9. closure of the gap and application of the polymerization pressure;

10. heat-assisted polymerization (see below).

During the entire polymerization period and testing, two infrared lamps illuminated the specimen from opposite directions, in order to increase its temperature and lower the time needed for polymerization of the adhesive. The black adhesive tape was applied with the aim of absorbing the light radiation thus increasing the adherends and adhesive temperature. A preliminary calibration showed that under these conditions, the specimen 
reached a steady temperature of about $45^{\circ} \mathrm{C}$. This constant value was due not only to the infrared lamps, but also to the heat dissipated by the hydraulic circuit of the testing machine.

The test procedure was organized in two steps: polymerization of the adhesive and torque loading of the specimen up to failure. In the first step, the desired contact pressure was reached applying the axial load at a rate of $5 \mathrm{kN} / \mathrm{min}$, which ensures that the contact pressure value is reached before polymerization of the adhesive is started. Hence, the axial load was kept constant for six hours. According to the manufacturer's datasheet, this time period, in combination with a curing temperature of $45^{\circ} \mathrm{C}$, makes possible a complete polymerization of the adhesive. Therefore, full mechanical properties [16]-[17] were obtained.

In the second step, the torque failure tests were performed. In case the same contact pressure as during polymerization was applied to the specimen, the test started immediately after the end of polymerization. While maintaining constant the axial force, a rotary motion at the ratio of $0.2^{\circ} / \mathrm{s}$ was applied to the upper adherend upon sweeping a $20^{\circ}$ angle. Contrarily, in the case a failure test had to be done at a different contact pressure than polymerization, the specimen was loaded or unloaded at a rate of $500 \mathrm{~N} / \mathrm{min}$ (while keeping a zero torque) till to the desired value. Such low value of loading rate aims at minimizing damages of the hybrid interface, in particular when the axial load decreases at the end of polymerization. Once the axial load corresponding to the chosen nominal pressure value was reached, it was kept steady on the specimen for two hours, in order to stabilize the mechanical response of the hybrid interface. This procedure was adopted as in Dragoni et 
al. [13]-[14], which observe that, after a contact pressure variation, the mechanical properties of both hybrid and purely friction interfaces slowly increase and become stable within a couple of hours. At the end of the stabilization, the torque failure test was performed as described above (a $20^{\circ}$ angle swept at a constant rate of $0.2^{\circ} / \mathrm{s}$ ).

In addition to this main experimental campaign, some tests were performed to assess the effect of a temporary reduction of the contact pressure on the shear strength of the hybrid interface. This is an important issue for machine components where sometimes preload can be temporarily released. Both the weak and strong anaerobic adhesives were tested, performing three replicates for each of them. The test procedure was as follows: polymerization of the adhesive under high nominal pressure level, unloading of the specimen up to the low nominal pressure value, and, finally, loading of the specimen up to the initial high nominal pressure value (see Table 1). Both unloading and loading were performed at the same rates described above, and each load level was kept constant two hours. The torque failure test was performed at the end of this procedure, again with a rotary motion speed equal to $0.2 \%$.

All these test procedures were implemented through the control software of the testing machine in order to make possible accurate replications, both with regard to the time period and to the load values and load ratio. According to the design of experiment criteria [18], the tests were performed in a randomized order. 


\section{Results}

The measured average roughness $\left(R_{a}\right)$ of the bonding surface of the adherends was equal to about $1.5 \mu \mathrm{m}$, with a standard deviation of about $0.3 \mu \mathrm{m}$. Figure 3 shows the typical area of contact of a specimen subject to an axial load of $1 \mathrm{kN}$ (Figure $3 \mathrm{a}$ ) and $24 \mathrm{kN}$ (Figure $3 \mathrm{~b}$ ). For each of the four experimental configurations investigated with Loctite 243, Figure 4 reports the diagram of the torque load applied to the specimen as a function of the rotation angle. Each diagram collects three curves, one for each of the replicates done for that configuration. Figure 5 shows the same diagrams for Loctite 638. Figure 6 reports analogous curves for the dry (unbonded) specimen configuration. Figure 7 displays pictures of the interface after failure of the specimen for Loctite 243 adhesive (Figure 7a), Loctite 638 adhesive (Figure 7b), and for a dry specimen (Figure 7c). For each configuration, Figure 7 displays a magnified detail (100x) of the failure surface, obtained through a digital optical microscope.

Figure 8 summarizes the values of the shear strength, $\tau_{R}$, as a function of applied pressure at the interface during test, $p_{\text {test }}$, both for Loctite 243 (Figure 8a) and for Loctite 638 (Figure $8 b)$. The shear strength was calculated as the ratio between the maximum torque load, $M_{t}$, and the equivalent polar section modulus, according to the following relationship:

$$
\tau=\frac{16 M_{t}}{\pi\left(D^{2}-d^{2}\right)(D+d)}
$$

where $D$ is the external diameter of the adherend and $d$ is the diameter of the inner hole. 
The diagrams in Figure 9 show the shear strength as a function of the applied pressure during polymerization, $p_{\text {polym }}$, as provided by the analysis of variance, done with the Design Expert commercial software [19]. The analysis of variance was performed independently for each adhesive: Loctite 243 (Figure 9a) and Loctite 638 (Figure 9b). The experimental data from Loctite 243 were transformed by applying the square root function, as suggested by the software. In Figure 9, the solid line and the dashed line identify, respectively, the response at the lower and higher level of applied pressure during test, $p_{\text {test }}$, while the solid circles correspond to the values from the experimental tests. 


\section{Discussion}

The average roughness value $\left(R_{a}\right)$ of the bonding area of the adherends together with its low standard deviation proves a high level of accuracy of the surface for the whole set of specimens. Moreover, Figure 3 highlights a uniform distribution of the contact area over the annular surface, both at the higher $(24 \mathrm{kN})$ and lower $(1 \mathrm{kN})$ load level. This result, which was observed for all the specimens, testifies an accurate machining of the specimen ends, thus fostering the achievement of good interface conditions.

The torque-angle curves in Figure 4 and Figure 5 show a marked repeatability of the results both for the weak (Loctite 243, Figure 4) and for the strong (Loctite 638, Figure 5) anaerobic. For each configuration investigated, the linear elastic portions of the three replications are in excellent agreement. Also, the maximum torque load and the post-elastic part of the curves- up to complete failure of the interface are quite close to each other. In particular, all the tests show a good stabilization of the post-elastic response, confirming that a complete failure of the interface occurred.

The same observations apply to the purely friction interface (Figure 6), where the only difference compared to the hybrid interface is a slight increase of the torque load as the rotation angle increases. This behaviour may be imputed to local overheating originated by friction between the contact surfaces that causes, on the micro-scale, local welding between contacting asperities. As a results, the global torque load increases since these local weldings have to be broken. 
The pictures in Figure 7a-b highlight a uniform distribution of the adhesive on the mating surfaces of the adherends. Moreover, the magnified views of the failure surfaces show a thin adhesive layer on both adherends, thus confirming that cohesive failure occurs. The thin adhesive layer has a more uniform thickness in case of the strong anaerobic (Loctite 638 , Figure $7 b$ ) than for the weak anaerobic (Loctite 243, Figure 7a). In the case of the weak anaerobic, moreover, some spots of the underlying metallic adherend are clearly visible. In the case of the purely friction specimen (Figure 7c) it is possible to observe the grooves originated by sandpaper abrasion in the initial preparation of the adherends. These grooves, in the case of hybrid interfaces, were probably filled by the adhesive (see magnified views in Figure 7a-b).

Figure 8 confirms the very low scatter of the experimental results for all the configurations investigated. By examining the four load configurations considered in the experimental plan, the following four observations can be drawn. First, when the contact pressure applied to the hybrid interface is low, both during polymerization and failure test, the shear strength of the adhesive (solid squares in Figure 8) equals about $5 \mathrm{MPa}$ for Loctite 243 and about 27 MPa for Loctite 638. These values agree with the standard strengths reported by the technical data sheets of these adhesives. Second, the shear strength increases significantly if the contact pressure applied to the hybrid interface is high both during polymerization and failure test (solid triangles in Figure 8). This loading condition determines an increase of the shear strength of 7 times for the weak anaerobic (Loctite 243) and of 2.5 times for the strong anaerobic (Loctite 243). Third, if the contact pressure is high during polymerization but low during failure testing, then the shear strength of the adhesive drops down to a 
nearly zero value (empty squares in Figure 8). This behaviour testifies that a decrease in the contact pressure after polymerization promotes the damaging of the hybrid interface. The cause of this damaging is probably the elastic spring back of the mating asperities that trigger micro-fractures in the adhesive layer interposed between the asperities, hence drastically reducing its strength. Fourth, for low contact pressure during polymerization and high pressure applied before the failure test, the adhesive exhibits a significantly high shear strength (empty triangles in Figure 8). In particular, the adhesive's shear strength is higher than the strength registered when the same contact pressure value is steadily applied to the hybrid interface since polymerization. This result corroborates the fundamental hypothesis of the micromechanical model (Appendix 1): a thin adhesive layer always remains between the roughness protrusions, increasing its shear strength under the high local pressure (equal to yield strength of the adherends). The higher shear strength compared to a condition where high contact pressure is applied before polymerization can be explained as follows. If the contact pressure is applied to the hybrid interface before polymerization of the anaerobic adhesive, only the thin adhesive layer which remains between mating surface protrusions bears the load, undergoing direct pressure. By contrast, if the contact pressure is applied to the hybrid interface when the anaerobic adhesive is polymerized (and hence solidified), the load is borne both by the adhesive lying between mating surface protrusions, and by the adhesive filling the voids between protrusions. Therefore, also this latter receives a share of direct pressure and, according to the micro-mechanical model (Appendix 1), increases its strength. 
The results from the specimen where contact pressure has been temporarily removed (crosses in Figure 8) show that the shear strength of the interface, significantly impaired by a release of the contact pressure (empty squares in Figure 8), is nearly completely restored by applying again the contact pressure. In particular, after reloading the interface reaches shear strength values close to those of the interface subject to constant contact pressure since polymerization (solid triangles in Figure 8). This result also strengthens the fundamental assumption of the micromechanical model (local stress of the adhesive improved by the local pressure, Appendix 1). The strong anaerobic adhesive still has shear strength higher than that of a dry friction interface.

The results from the pure friction interface (empty circles Figure 8) highlight a friction coefficient equal to 0.3 , in accordance with the results provided in [10] and typical of a non-lubricated steel to steel contact. A comparison between the data from the hybrid interface and the results from the pure friction interface (empty circles Figure 8), highlights contradictory effects. On the one hand, it appears that the weak anaerobic (Loctite 243), under a steady high contact pressure since polymerization (solid triangles), causes a slight decrease of the average shear strength with respect to the pure friction interface. This could be explained as a sort of lubricant effect introduced by the weak adhesive with respect to a pure friction contact between metal crests of the surfaces. By contrast, in case a low contact pressure is steadily applied since polymerization (solid squares in Figure 8), the weak adhesive increases the shear strength of the interface. For the same weak adhesive, if the contact pressure is increased at the end of polymerization (hollow triangles), then the hybrid interface exhibits a higher shear strength compared to a pure friction interface. On 
the other hand, the strong anaerobic (Loctite 638) significantly increases the shear strength of the interface on the whole range of contact pressure here examined.

All the above observations are confirmed by the diagrams in Figure 9. The analysis of variance clearly assesses that for both adhesives the shear strength first of all depends on the contact pressure both during polymerization and during failure test. In addition, the interaction between these variables also affects shear strength as shown by the convergent lines in the diagrams in Figure 9. Moreover, the diagrams highlight that by increasing the contact pressure after polymerization, a maximum value of the shear strength of the hybrid interface is obtained.

On the whole, these observations confirm the applicability of the micro-mechanical model proposed in [14] by Dragoni et al:: a thin adhesive layer always remains between the roughness protrusions, increasing its shear strength under the high local pressure (equal to the yield strength of the adherends). This micro-mechanical model was recently put to test also through a computational analysis campaign which modelled the adhesive in terms of this same micro-mechanical model [20]. The results of the computational analysis favourably confirmed the model. 


\section{Conclusions}

The work investigates the static shear strength of hybrid friction-bonded interfaces through an experimental campaign including three variables over two levels. The tests examine the static shear strength of tubular specimens, butt-bonded and undergoing a uniform normal and shear stress field. In order to have a comparison, the shear strength of a dry specimen was also examined. The results highlight that:

- the static shear strength of the hybrid specimens increases with contact pressure, similarly to the dry (purely friction) specimens;

- the strong anaerobic adhesive significantly improves the shear strength of the interface on the whole pressure range here examined;

- the weak anaerobic adhesive, by contrast, improves the shear strength of the interface only only when the contact pressure is low;

- regardless of the adhesive type, polymerization at low contact pressure followed by testing at high pressure provides higher shear strength of the interface than a constantly high pressure since the beginning of polymerization;

- a release of the contact pressure after polymerization causes damage of the interface thus lowering its shear strength;

- restoring the contact pressure (on the above specimens), the shear strength of the interface is nearly completely recovered; 
- on the whole, the results confirms the applicability of the micro-mechanical model (Appendix 1) proposed by Dragoni et al [13]. A thin adhesive layer always remains between the roughness protrusions, increasing its shear strength under the high local pressure (equal to yield strength of the adherends). 


\section{References}

[1] O’Reilly, C., in Proc. of SAE International Congress and Exposition, Detroit, Michigan, paper 900776 (1990).

[2] Mahon, F., in SAE International Congress and Exposition, Detroit, Michigan, paper 950125 (1995).

[3] Romanos, G., Int. J. Mater. Product Technol. 14, 430-443 (1999).

[4] Bartolozzi, G., Croccolo, D., and Chiapparini, M, OIAZ 144, 198-201 (1999).

[5] Croccolo, D., De Agostinis, M., and Vincenzi, N., Int. J. of Adhes. Adhes. 30, 359-366 (2010).

[6] Sekercioglu, T., Gulsoz, A., and Rende, H., Mater. Des. 26, 377-81 (2005).

[7] Sekercioglu, T., Int. J. Adhes. Adhes. 25, 352-7 (2005).

[8] Sekercioglu, T., and Meran, C., Mater. Des. 25, 171-175 (2004).

[9] Aronovich, D.A., Murokh, A.F., Sineokov, A.P., and Khamidulova, Z.S., Polymer Science. 1, 260-265 (2008).

[10]Mengel, R., Häberle, J., and Schlimmer, M., Int. J. of Adhes. Adhes. 27, 568-573 (2007).

[11] Oinonen, A., and Marquis, G., Int. J. Adhes. Adhes. 31, 550-558 (2011).

[12] Oinonen, A., and Marquis, G., Engng. Fracture Mech. 78, 163-174 (2011).

[13]Dragoni, E., and Mauri, P., Int. J. Adhes. Adhes. 20, 315-321 (2000).

[14]Dragoni, E., and Mauri, P., Proc. Instn. Mech. Engrs., Part L 216, 9-15 (2002).

[15]Halling, J., Introduction to tribology, (Wykeham Engineering and Technology Series, London, 1976). 
[16]Loctite 243, TDS, http://65.213.72.112/tds5/docs/243-IT.PDF (2009).

[17]Loctite 638, TDS, http://65.213.72.112/tds5/docs/638-IT.PDF (2009).

[18] Montgomery, D.C., (John Wiley and Sons, 2005).

[19] StatEase, Design Expert User's Manual, Minneapolis, USA, 2005.

[20] Castagnetti, D., Dragoni, E., J. of Comp. Mat. Sci. 64, 146-150 (2012).

[21]Raghava, R.S., Cadell, R.M. J. Mater. Sci. 8, 225-232 (1973).

[22] Schlimmer, M., Werkstofftech, 13, 215-221, (2004).

[23] Mengel, R., Häberle, J., Schlimmer, M., Int. J. of Adhes. Adhes. 27, 568-573 (2007). 


\section{Appendix 1}

The simple micromechanical model put forward in [14] assumes that the two rough surfaces touch each other over a fraction, $A_{r}$ (real area of contact), of the nominal contact area, $A$ (Figure A1a). It is also assumed that the adhesive fills the voids all around the protrusions, where it receives no pressure, and forms a thin layer between them. This interlayer is subjected to the yield pressure, $Y$, of the softest adherend. The yield pressure $Y$ is a constant once the adherend materials have been chosen.

In order to break the joint, the area, $A_{e}=A-A_{r}$, of the adhesive around the asperities and the real contact area, $A_{r}$, of the adhesive in between must be fractured simultaneously. If $\tau_{a o}$ is the unit shear strength of the adhesive at zero pressure and $\tau_{a Y}$ the shear strength of the adhesive under a pressure equal to $Y$, the shear failure load, $T$, of the joint amounts to $T=$ $\tau_{a o} A_{e}+\tau_{a Y} A_{r}=\tau_{a o}\left(A-A_{r}\right)+\tau_{a Y} A_{r}$. From the equilibrium condition for each adherend $\left(Y A_{r}=P\right)$ the real area of contact is calculated as $A_{r}=P / Y$, which is proportional to the load $P$. Combination of the expressions for $T$ and $A_{r}$ gives:

$$
T=\tau_{a o} A+\left(\tau_{a Y}-\tau_{a o}\right) P / Y=T_{o}+T_{Y}
$$

Equation (1) predicts the macroscopic strength of the joint as the sum of a constant term, $T_{o}=\tau_{a o} A$, and a variable term, $T_{Y}=\left(\tau_{a Y}-\tau_{a o}\right) P / Y$, which is proportional to the clamping force. For a dry joint, in which $\tau_{a o} \equiv 0$ and $\tau_{a Y} \equiv \tau_{Y}=$ shear strength of the metal junctions, 
the above formula predicts $T_{o}=0$ and $T_{Y}=\tau_{Y} P / Y$ in accordance with Coulomb law. For a bonded joint where the adhesive would be squeezed out of the junctions upon tightening $\left(\tau_{a Y} \equiv \tau_{Y}>>\tau_{a o}\right)$, Eq. (A1) would predict the same constant strength $T_{o}=\tau_{a o} A$ as in the purely adhesive joint together with a pressure-dependent strength $T_{Y}=\left(\tau_{Y}-\right.$ $\left.\tau_{a o}\right) P / Y \approx \tau_{Y} P / Y$ with the same slope as the dry joint. This is the rationale behind the criterion of superimposition of effects, stated in the Introduction, that must be dismissed on experimental grounds.

A possible explanation of the experimental results [14] is that a thin film of anaerobic is actually retained between the crests of the clashing surfaces. Similarly to what happens for regular polymers [21]-[23], under the high local pressure $(Y)$ this film can attain a shear strength $\left(\tau_{a Y}\right)$ significantly greater than the shear strength at zero pressure $\left(\tau_{a o}\right)$. Accordingly, one would expect that the higher the strength at zero pressure $\left(\tau_{a o}\right)$, the higher the strength $\left(\tau_{a Y}\right)$ under the yield pressure of the adherends and the higher the slope, $\left(\tau_{Y}-\tau_{a o}\right) / Y$, of the characteristic curve in response to the tightening load (Figure Alb). These assumptions can explain the crossing of the curves for hybrid and dry interfaces observed in [14]. 


\section{Figures and Tables caption}

Figure 1 Detail drawing of the specimen (a, dimensions in $\mathrm{mm}$ ) and picture of a test piece (b) seen from the bonding end.

Figure 2 Set up of the specimen on the testing machine.

Figure 3 Picture of the contact surface for a $1 \mathrm{kN}$ (a) and $24 \mathrm{kN}$ (b) applied load.

Figure 4 Experimental curves of torque load against twist angle for Loctite 243 (three replicates).

Figure 5 Experimental curves of torque load against twist angle for Loctite 638 (three replicates).

Figure 6 Experimental curves of torque load against twist angle for dry specimens (three replicates).

Figure 7 Pictures of failed specimens with magnification (100x) of the interface features: Loctite 243 (a), Loctite 638 (b), and dry specimen (c). 
Figure 8 Failure shear stress, $\tau_{R}$, as a function of the applied pressure during test, $p_{\text {test }}$ :

(a) Loctite 243, and (b) Loctite 638 (the hollow circles represent the purely friction interface).

Figure 9 Interaction plot for the shear strength, $\tau_{R}$, as a function of the pressure during polymerization, $p_{\text {polim }}$, and the pressure during test, $p_{\text {test }}$ (a) Loctite 243 , (b) Loctite 638. Solid circles corresponding to the single experimental tests.

Figure A1. Micromechanical model of the hybrid interface (a) and typical predictions for its macroscopic shear strength (b).

Table 1 Variables and levels for the designed experiment. 


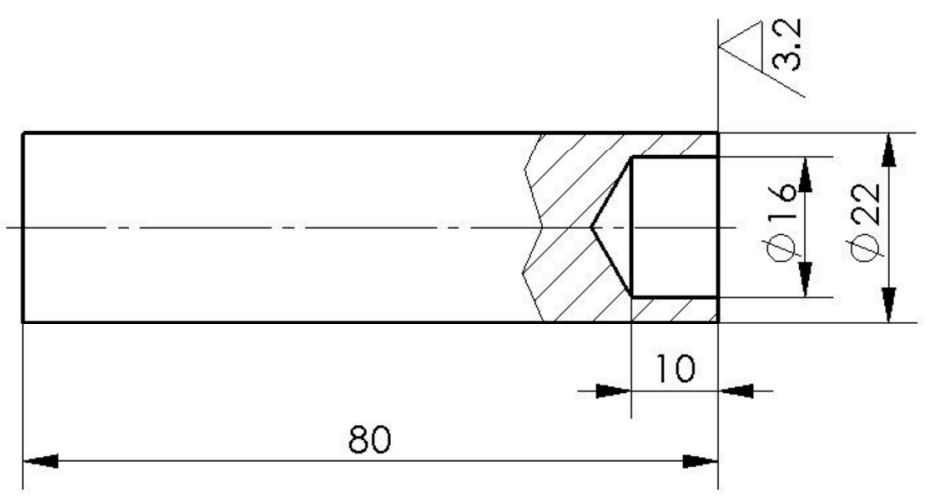

(a)

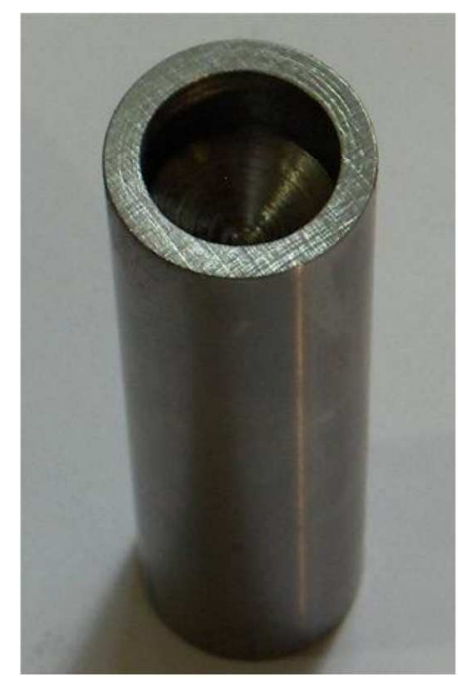

(b)

\section{Figure 1}

$97 \times 160 \mathrm{~mm}(600 \times 600 \mathrm{DPI})$

URL: http://www.tandfonline.com/gadh Email: lucas@fe.up.pt 


\section{Figure 2}

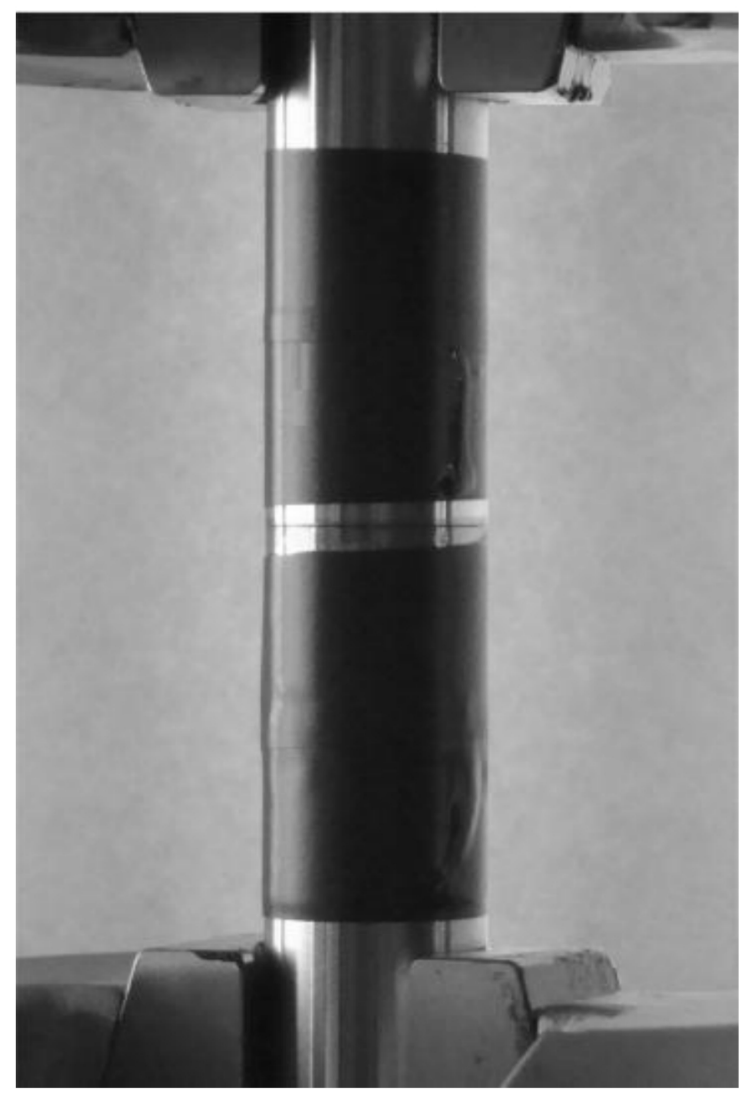

$71 \times 143 \mathrm{~mm}(600 \times 600 \mathrm{DPI})$ 
(a)

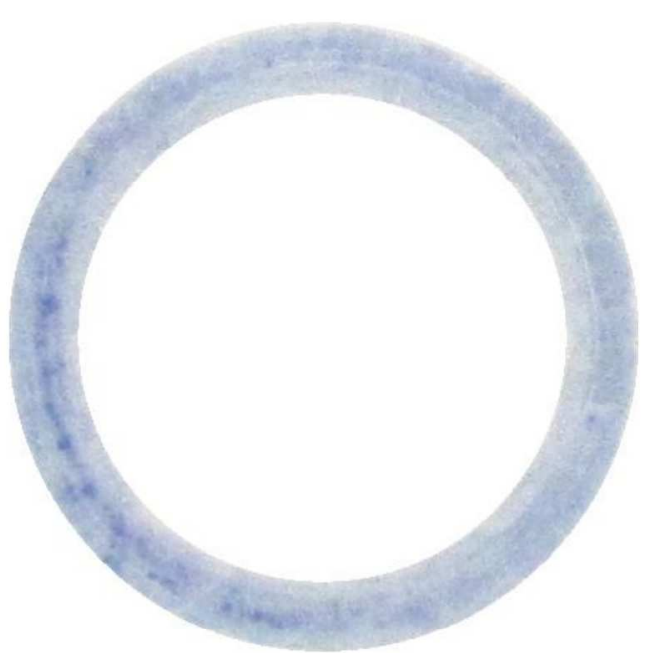

(b)

Figure 3

$142 \times 293 \mathrm{~mm}(600 \times 600 \mathrm{DPI})$

URL: http://www.tandfonline.com/gadh Email: lucas@fe.up.pt 

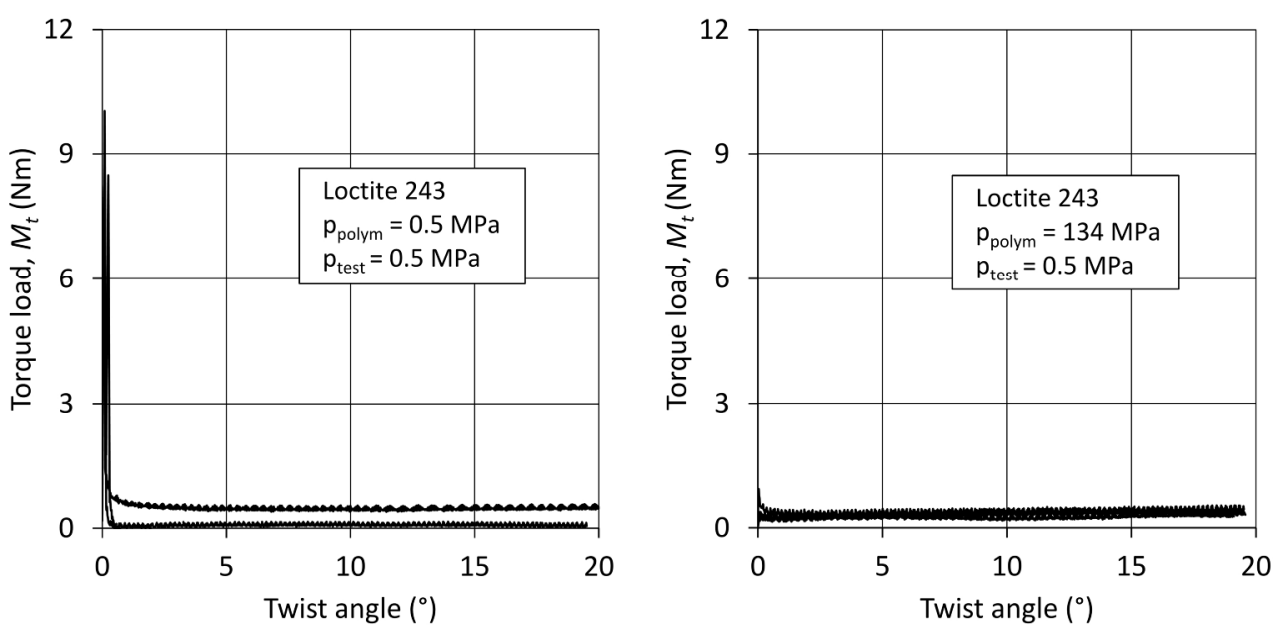

(a)

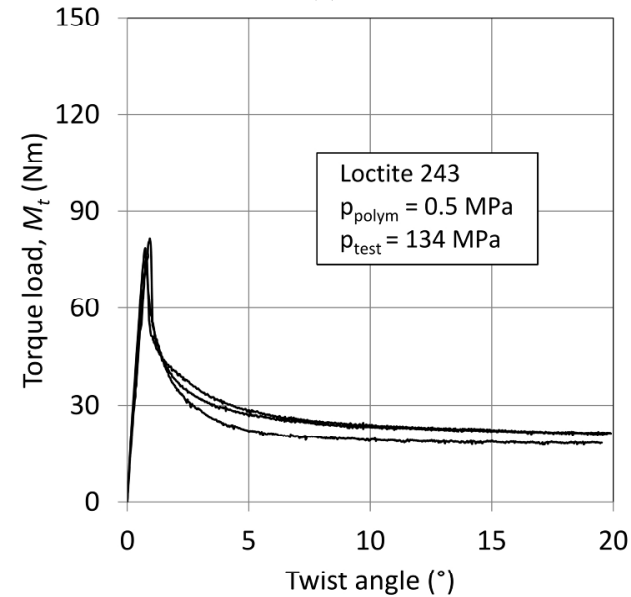

(c)

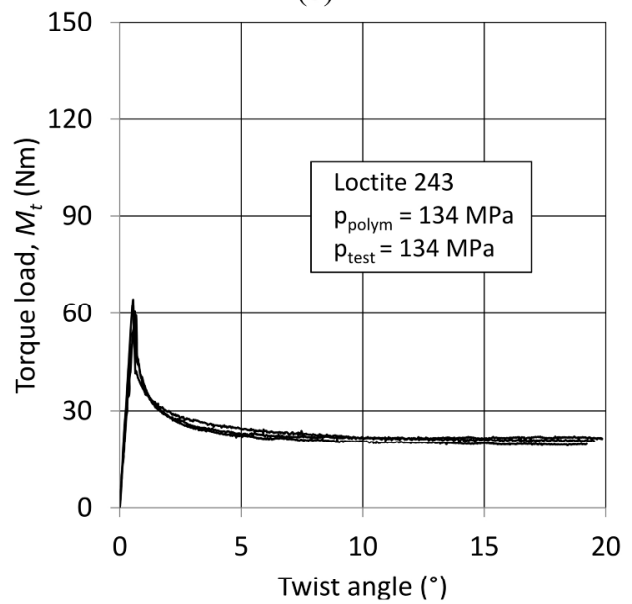

(d)

Figure 4

$127 \times 134 \mathrm{~mm}(600 \times 600 \mathrm{DPI})$

URL: http://www.tandfonline.com/gadh Email: lucas@fe.up.pt 


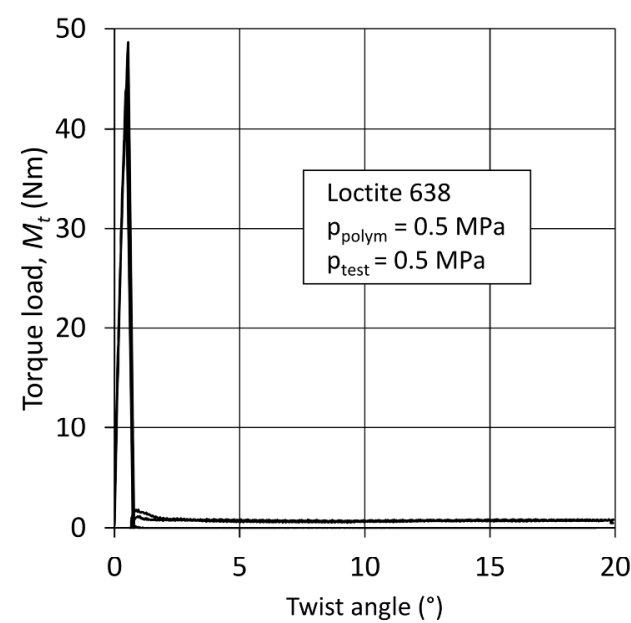

(a)

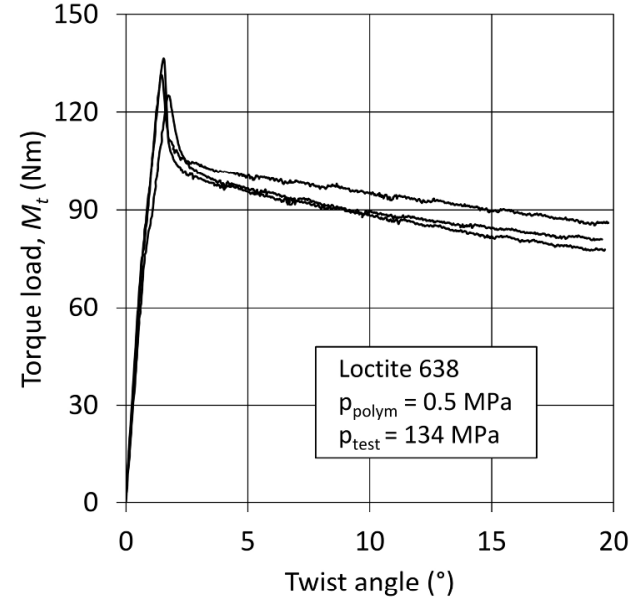

(c)

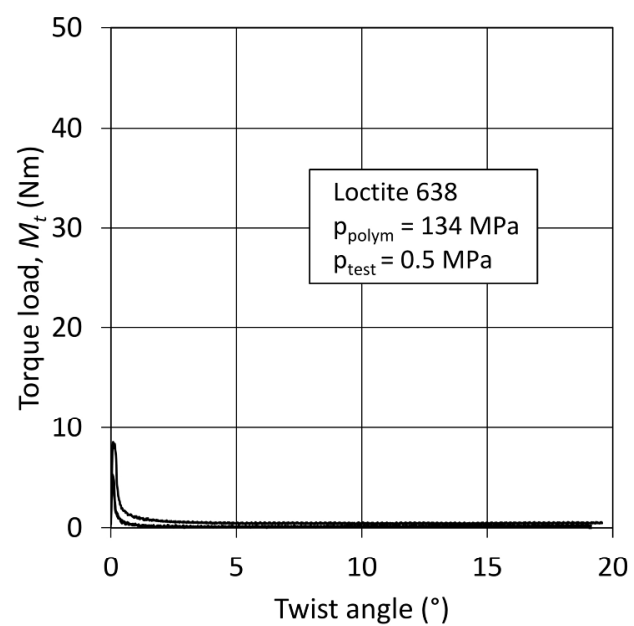

(b)

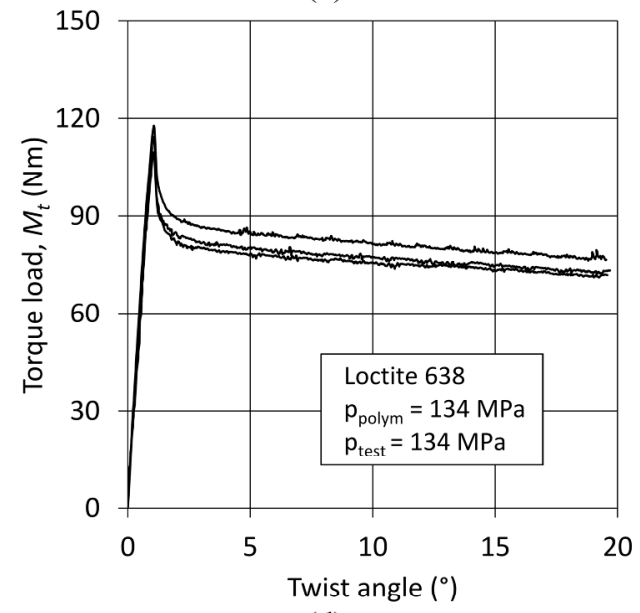

(d)

Figure 5

$128 \times 137 \mathrm{~mm}(600 \times 600 \mathrm{DPI})$

URL: http://www.tandfonline.com/gadh Email: lucas@fe.up.pt 


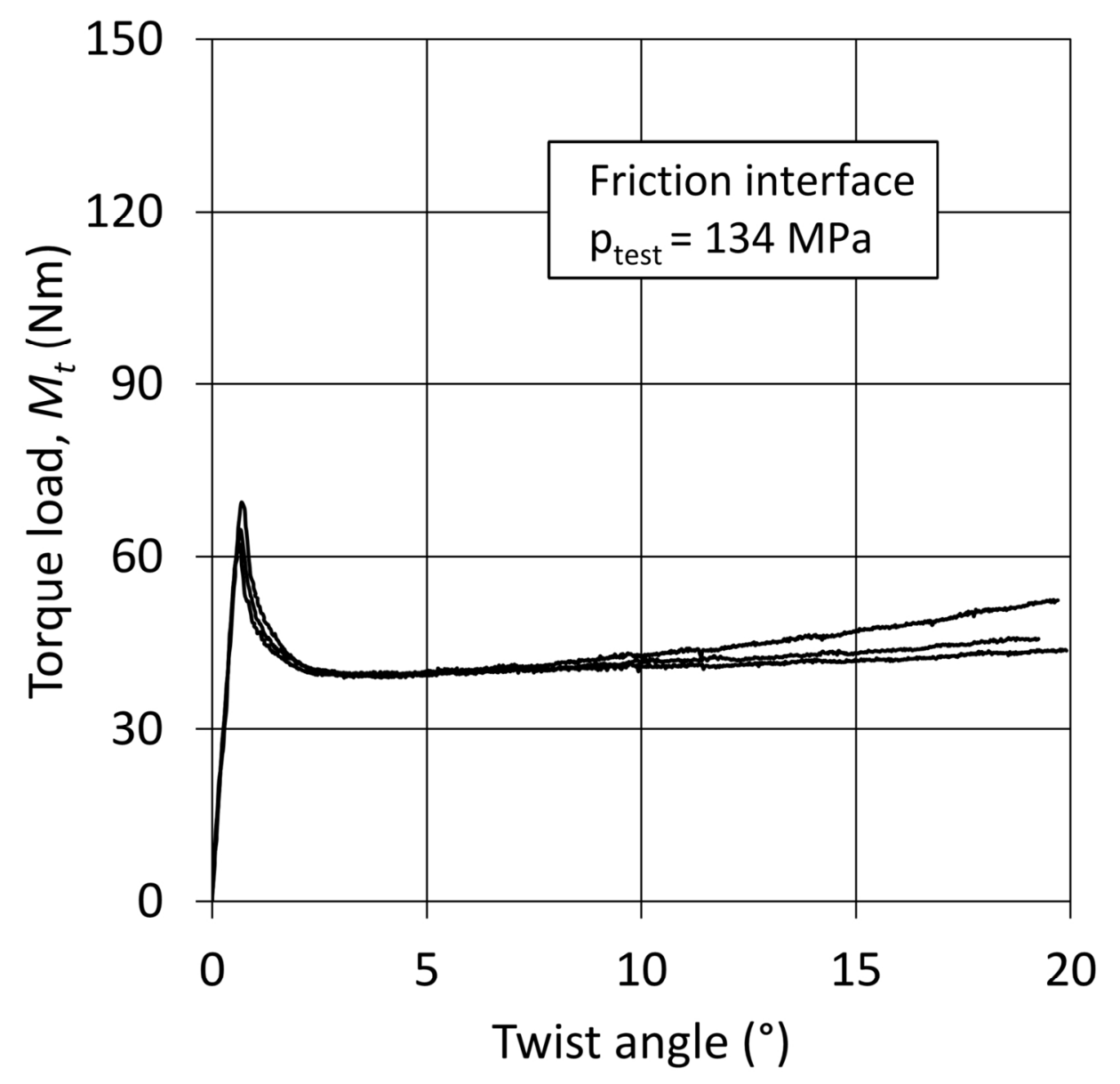

Figure 6

$72 \times 88 \mathrm{~mm}(600 \times 600 \mathrm{DPI})$

URL: http://www.tandfonline.com/gadh Email: lucas@fe.up.pt 


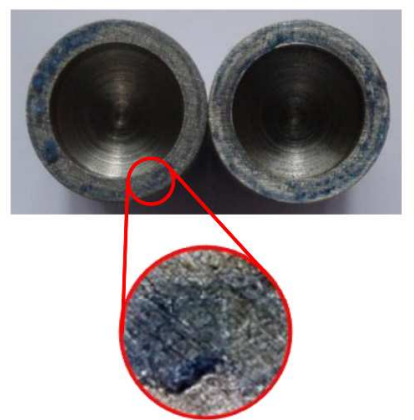

(a)

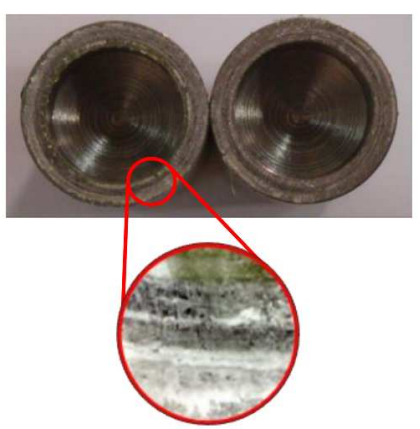

(b)

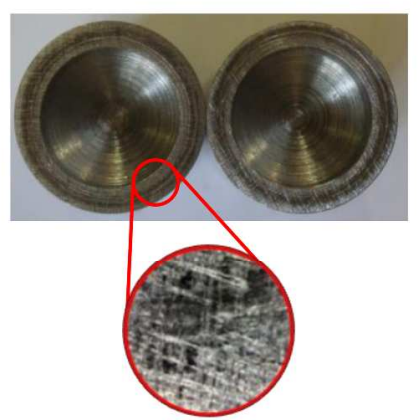

(c)

Figure 7

$144 \times 419 \mathrm{~mm}(600 \times 600 \mathrm{DPI})$

URL: http://www.tandfonline.com/gadh Email: lucas@fe.up.pt 


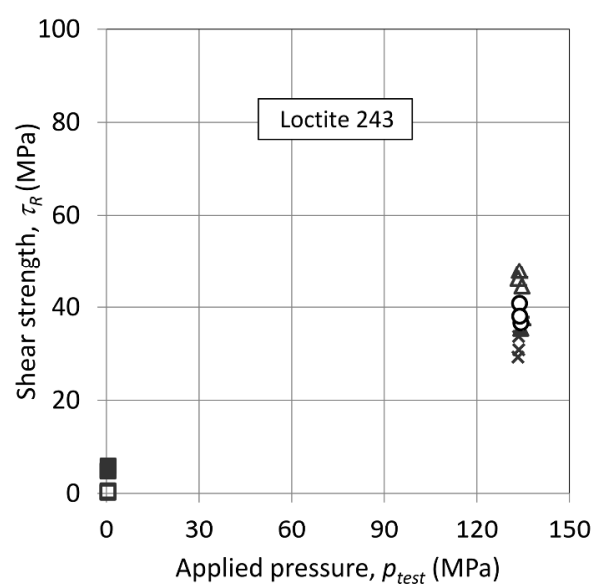

(a)

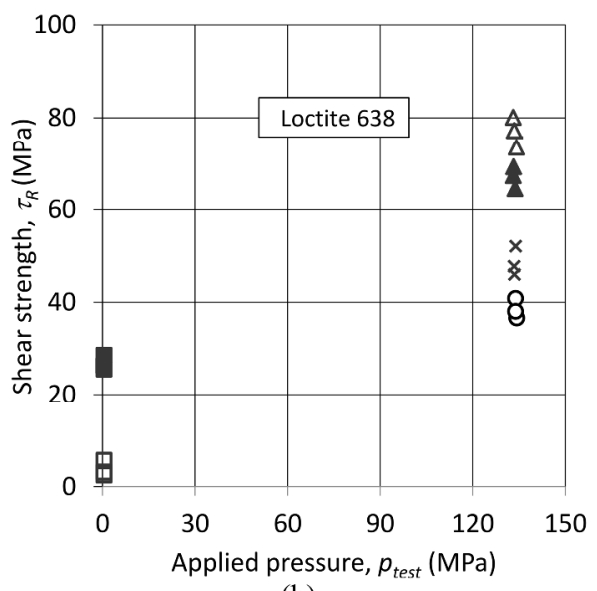

(b)

$$
\begin{aligned}
& -p_{\text {polym }}=0.5 \mathrm{MPa}-p_{\text {test }}=0.5 \mathrm{MPa} \\
& \Delta p_{\text {polym }}=0.5 \mathrm{MPa}-p_{\text {test }}=134 \mathrm{MPa} \\
& \Delta p_{\text {polym }}=134 \mathrm{MPa}-p_{\text {test }}=134 \mathrm{MPa} \\
& \square p_{\text {polym }}=134 \mathrm{MPa}-p_{\text {test }}=0.5 \mathrm{MPa} \\
& \times p_{\text {polym }}=134 \mathrm{MPa}-p_{\text {temp }}=0.5 \mathrm{MPa}-p_{\text {test }}=134 \mathrm{MPa} \\
& \circ p_{\text {test }}=134 \mathrm{MPa} \text { (dry friction interface) }
\end{aligned}
$$

Figure 8

$$
155 \times 379 \mathrm{~mm}(600 \times 600 \mathrm{DPI})
$$




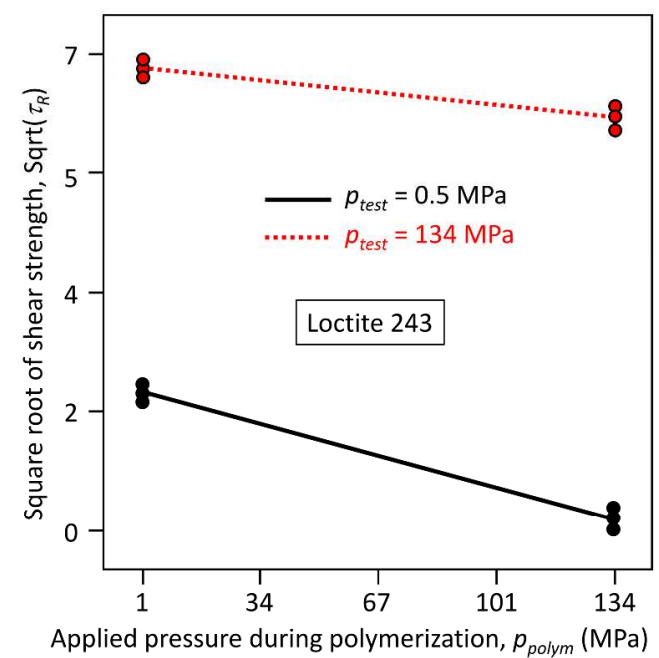

(a)

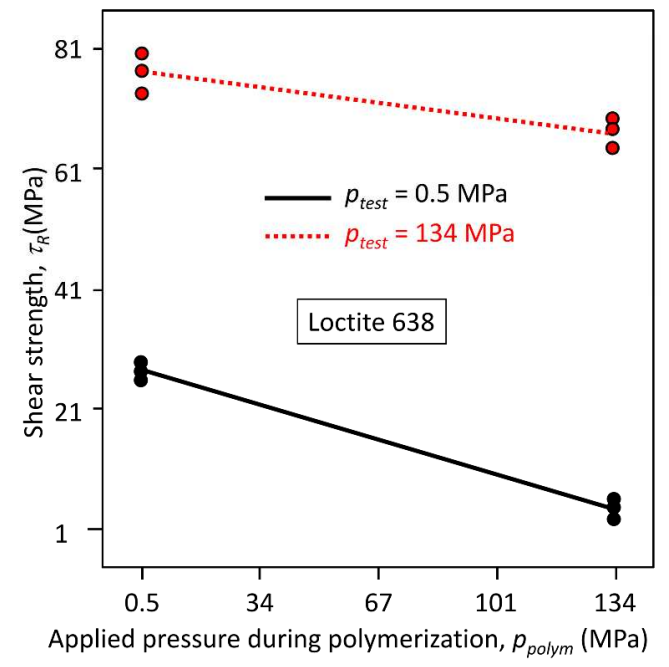

(b)

Figure 9

$136 \times 307 \mathrm{~mm}(600 \times 600 \mathrm{DPI})$

URL: http://www.tandfonline.com/gadh Email: lucas@fe.up.pt 


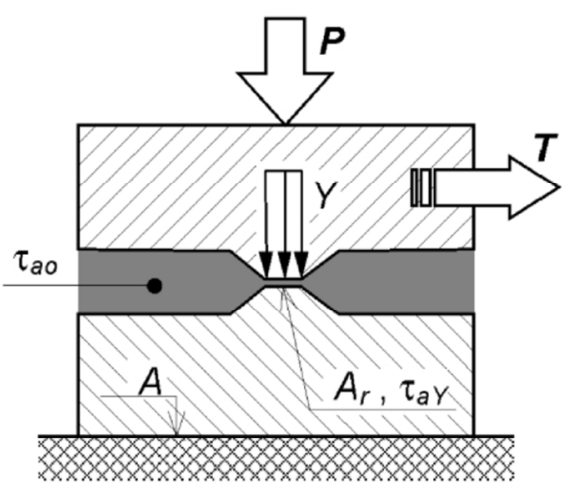

(a)

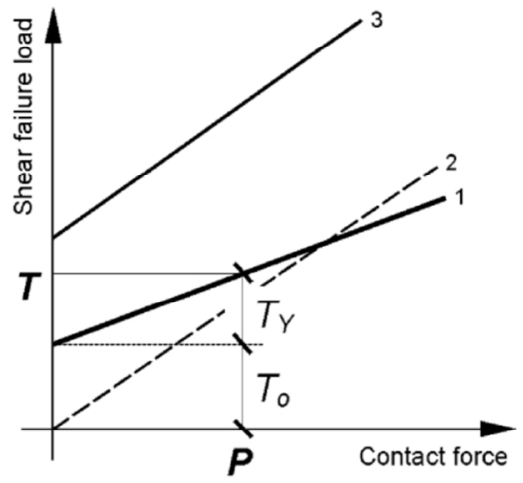

(b)

Figure A1.

$57 \times 34 \mathrm{~mm}(600 \times 600 \mathrm{DPI})$

URL: http://www.tandfonline.com/gadh Email: lucas@fe.up.pt 
Table 1

\begin{tabular}{lcc}
\hline \multicolumn{1}{c}{ Variable } & - & + \\
\hline Adhesive & Loctite 243 & Loctite 638 \\
\hline $\begin{array}{l}\text { Nominal pressure during polymerization, } p_{\text {polym }} \\
(\mathrm{MPa})\end{array}$ & 0.5 & 134 \\
\hline Nominal pressure during failure test, $p_{\text {test }}(\mathrm{MPa})$ & 0.5 & 134 \\
\hline
\end{tabular}

$29 \times 8 \mathrm{~mm}(600 \times 600 \mathrm{DPI})$

URL: http://www.tandfonline.com/gadh Email: lucas@fe.up.pt 